\title{
The effectiveness of an integrated dietary intervention compared with an average UK diet in reducing cardiovascular disease risk factors in older men and women aged 40 to 70 years: the CRESSIDA Study
}

\author{
D. P. Reidlinger ${ }^{1}$, J. Darzi ${ }^{1}$, W. L. Hall ${ }^{1}$, Z. Maniou ${ }^{1}$, V. Govoni ${ }^{1}$, P. Seed ${ }^{2}$, P. J. Chowienczyk ${ }^{3}$ \\ and T. A. B. Sanders ${ }^{1}$ \\ ${ }^{1}$ Diabetes and Nutritional Sciences Division, King's College London, 150 Stamford Street, London SE1 9NH, UK, ${ }^{2}$ Division \\ of Women's Health, and ${ }^{3}$ Cardiovascular Division, King's College London, St Thomas' Hospital, London SE1 7EH, UK
}

Dietary guidelines ${ }^{(1,2)}$ for the prevention of cardiovascular disease have not been systematically evaluated in a randomized controlled trial. We carried out a parallel designed randomized controlled trial comparing a cardioprotective diet with a traditional British dietary pattern in healthy non-smoking men and women aged $40-70$ years. The cardioprotective diet included salt $<6$ g/day, saturated fatty acids $<10 \%$ of food energy, oily fish intake 1-2 portions/week and fruit and vegetables 5 portions/day, and supplied at least half of the cereal intake from wholegrains. The control diet was a typical well-balanced British diet containing less than one serving per month of oily fish. Following a screening visit, potential participants were asked to make $2 \times 24$ hour urine collections, $2 \times 24$ hour ambulatory blood pressure (BP) measurements and complete a 4-day dietary record. Participants attended a clinic to have specialised measurements of vascular function made and to provide fasting blood samples, and were then randomly allocated to the diets. Dietary advice, given by a dietitian, was followed up at regular intervals and a variety of foods were provided to aid compliance to the diet. Further 24 hour BP monitoring and urine collections were made at 4-6 weeks, 8 weeks and 12 weeks and further dietary assessments, vascular measurements and blood collections were made at the end of the study. The specified primary outcomes reported here were a $4 \mathrm{~mm} \mathrm{Hg}$ change in daytime systolic BP and a 5\% change in the ratio of total cholesterol:HDL cholesterol (TC:HDL-C). Data were analysed on an intention to treat basis by analysis of covariance adjusting for the baseline value, age, gender, body mass index and ethnicity using STATA version 11.1 (StataCorp, College Station Texas). The CRESSIDA trial (ISRCTN92382106) randomised 165 participants to either the cardioprotective (CP) or a control (C) diet for 12 weeks. Data for the primary outcomes were available for analysis in 162 subjects.

\begin{tabular}{|c|c|c|c|c|c|c|c|c|}
\hline & \multicolumn{4}{|c|}{ Daytime systolic BP $\mathrm{mm} \mathrm{Hg}$} & \multicolumn{4}{|c|}{ TC:HDL-C } \\
\hline & \multicolumn{2}{|c|}{$\mathrm{CP}(n 80)$} & \multicolumn{2}{|c|}{$\mathrm{C}(n \mathrm{82})$} & \multicolumn{2}{|c|}{$\mathrm{CP}(n 80)$} & \multicolumn{2}{|c|}{$\mathrm{C}(n 82)$} \\
\hline & Mean & SD & Mean & SD & Mean & $\overline{\mathrm{SD}}$ & $\overline{\text { Mean }}$ & $\overline{\mathrm{SD}}$ \\
\hline Baseline & 124.7 & 13.1 & 127.4 & 12.1 & 3.46 & 0.91 & 3.63 & $\overline{0.98}$ \\
\hline Follow-up & $122.3 * *$ & 13.5 & 128.7 & 13.1 & $3.31 *$ & 0.87 & 3.59 & 0.96 \\
\hline
\end{tabular}

$* P<0.05, * * P=0.001$ compared to control group

Day-time systolic BP fell by $4.2 \mathrm{~mm} \mathrm{Hg}(95 \%$ CI 1.7, 6.7) and the TC:HDL-C by $4.2 \%$ (95\% CI 0.2, 8.2). These changes are estimated to reduce the risk of fatal heart attack and stroke by $17 \%$ and $20 \%$ and incident cardiovascular disease by about a third. The dietary intervention was well accepted by the participants and provides evidence to support current dietary guidelines for the prevention of CHD.

This work was supported by the Food Standards Agency and the Department of Health.

1. Department of Health (1994) Nutritional Aspects of Cardiovascular Disease. Report on Health and Social Subjects No 46. London: HMSO.

2. National Institute for Health and Clinical Excellence (2010) Cardiovascular Disease Prevention. PHG 25. http://guidance.nice.org.uk/PH25 (accessed March 2013). 